\title{
Alzheimer's Disease: Memory Interference and the Role of Exercise
}

\author{
Lindsay Crawford • Paul D. Loprinzi \\ Exercise \& Memory Laboratory, Department of Health, Exercise Science, and Recreation \\ Management, The University of Mississippi, University, MS, USA
}

Author for correspondence: Paul D. Loprinzi, Exercise \& Memory Laboratory, Department of Health, Exercise Science, and Recreation Management, The University of Mississippi, University, MS 38677, USA. Email: pdloprin@olemiss.edu

Doi: http://dx.doi.org/10.15586/alzheimersdisease.2019.ch12

\begin{abstract}
Alzheimer's disease is an irreversible, progressive brain disorder that damages memory, behavioral, and cognitive skills. This condition causes brain cells to degenerate and die leading to many cognitive issues. Although the exact cause is unknown, it is thought to be due to a combination of genetic, lifestyle, and environmental factors. Due to its progressive nature, symptoms can vary from mild memory loss to complete lack of ability to respond to one's surroundings. The memory impairments brought on by this disease can lead to specific problems with memory interference, which may be caused by dysfunction in working and semantic memory. When conducting experiments on Alzheimer's patients, there is also the added difficulty of the individual having trouble remembering the instructions and needing external cues to complete memory tasks. This chapter outlines the disease, its symptoms, risk factors, how it affects memory, and how exercise may be a prevention and treatment option.
\end{abstract}

Keywords: Alzheimer's disease; memory; memory interference; proactive interference; retroactive interference

In: Alzheimer's Disease. Thomas Wisniewski (Editor), Codon Publications, Brisbane, Australia. ISBN: 978-0-646-80968-7; Doi: http://dx.doi.org/10.15586/alzheimersdisease.2019

Copyright: The Authors.

License: This open access article is licensed under Creative Commons Attribution 4.0 International (CC BY 4.0). https://creativecommons.org/licenses/by-nc/4.0/ 


\section{INTRODUCTION}

Alzheimer's disease is an irreversible, progressive brain disorder which damages memory, behavioral, and cognitive skills (1-4). This condition causes brain cells to degenerate and die, leading to many cognitive issues (4). It is the most common cause of dementia, accounting for 60 to $80 \%$ of dementia cases (2). Although the exact cause is unknown, it is thought to be due to a combination of genetic, lifestyle, and environmental factors (2). Due to its progressiveness, there are different signs and symptoms throughout the stages $(1,5)$. Typically, Alzheimer's disease progresses through stages, including mild (early stage), moderate (middle stage), and severe (late stage) (6). Since the condition affects people in different ways, individuals may have a unique experience to each stage. In the early stage, an individual may experience mild memory loss or difficulty remembering newly learned information, whereas in the late stage, individuals may lose the ability to function independently or respond to their surroundings $(2,5)$. Early-stage signs include forgetting names, appointments, recent events, trouble following instructions, keeping track of responsibilities (e.g., remembering to pay the bills), or difficulty using familiar items in their home (e.g., how to operate the thermostat) $(1,2,4-7)$. The middle stage is usually the longest stage in the disease progression and involves more serious signs of the first stage (6). An individual may have greater difficulty performing certain tasks and be more likely to become frustrated or withdrawn due to memory impairments (6). Signs of middle stage Alzheimer's disease include repeating statements or questions, routinely misplacing objects, getting lost in familiar places, losing track of the passing of time (e.g., forgetting which season it is), decreased or poor judgment, withdrawal from work or social activities, or changes in mood and personality (1, 2, 4-6). Personality and behavior changes often include depression, apathy, social withdrawal, mood swings, distrust, irritability, aggression, wandering, or delusions (4). Late stage is the final stage of Alzheimer's disease. The dementia symptoms are severe and individuals lose the ability to function independently or to react appropriately to their environment (6). During this stage, individuals may also lose their ability to control their motor movement and they will likely experience severe personality changes (6). At this stage, it is likely that the individual will need a full-time caregiver (6). Late-stage patients also become vulnerable to infections and other conditions due to memory impairment (e.g., dehydration, malnourishment) (6). Alzheimer's disease is the sixth leading cause of death in the United States, and on average, individuals live for 4 to 8 years after diagnosis (1). It is possible for people with Alzheimer's disease to live as long as 20 years post-diagnosis, but it is less common since it mostly occurs in individuals who are already 65 years or older (1).

\section{RISK FACTORS}

The greatest known risk factors for developing Alzheimer's disease is increasing age, as most individuals with Alzheimer's disease are 65 years or older (2). Another risk factor is family history (8). Individuals that have parents or siblings that have developed Alzheimer's disease are more likely to develop the disease $(4,8)$. 
The risk increases as members of the family with a diagnosis increase. Genetics also play a role in developing Alzheimer's themselves $(4,8)$. Genes for developing Alzheimer's disease have been found to be both deterministic and risk genes, meaning that they both cause the disease and increase the risk of developing the disease $(4,8)$. The deterministic gene, which almost guarantees the individual will develop the disease, only occurs in less than $1 \%$ of the Alzheimer's cases (4). Experiencing a head injury is also a possible risk factor for Alzheimer's disease (8). The most effective ways to prevent head injury are wearing seatbelt in any motor vehicle, wearing helmets, and "fall-proofing" homes for the elderly (8). An example of fall-proofing is placing non-slip mats in strategic areas of the home, especially in the bathroom and in the shower.

Sex, a common risk factor for many diseases (e.g., breast cancer), is not a risk factor for Alzheimer's disease (8). More women than men are diagnosed with Alzheimer's disease mainly due to the fact that women live longer than men making them more likely to develop Alzheimer's disease (8). Race may also play a role. Latinos and African Americans are more likely than Whites to develop Alzheimer's disease. This is not well understood, but it may be due to their increased rates of vascular disease $(4,8)$. Heart health and brain health are directly related, as dementia is associated with conditions that damage the heart and blood vessels (e.g., heart disease, stroke, high blood pressure, and high cholesterol) $(4,8)$. There is also evidence that plaques and tangles (physical evidence of Alzheimer's disease in the brain) are more likely to cause symptoms of dementia if damage to the brain's blood vessels is also apparent $(4,8)$.

Other lifestyle risk factors include lack of exercise, obesity, smoking, and poorly controlled type 2 diabetes $(4,8)$. All of these conditions are related to poor heart and brain health and can be modified by living a healthy active lifestyle that focuses on a whole food plant based diet (8). The Physician's Committee for Responsible Medicine recommends exercising regularly, limiting saturated and trans fats, eating plant based foods, eating foods rich in vitamin E, and taking a B12 supplement daily to prevent Alzheimer's disease (9). There is an increased risk for developing Alzheimer's disease in individuals with Down syndrome, which is most likely due to having three copies of chromosome 21 , which also includes having three copies of the gene that is associated with Alzheimer's disease (8). Symptoms of Alzheimer's disease usually appear 10 to 20 years earlier in people with Down syndrome compared to individuals without it (8).

\section{EARLY-ONSET ALZHEIMER'S DISEASE}

When the condition occurs in individuals younger than 65 , it is referred to as early-onset Alzheimer's disease (2). Early-onset Alzheimer's disease is less common, making up less than $5 \%$ of the population with Alzheimer's disease (around 200,000 people in the United States), and primarily affects people in their 40s and 50s (3). Since memory complications are less common in those under 65, it can be difficult to diagnosis early-onset Alzheimer's disease. The cause of early-onset is unclear, but there have been a few rare inherited genes that may play a factor in symptoms developing as early as 30 years old, referred to as "familial Alzheimer's disease" (3). 


\section{TREATMENT}

There is no cure for Alzheimer's disease, but treatments for symptoms are available as research into the condition continues (2). These drug or non-drug treatments may relieve some symptoms or slow the rate of mental decline. Their goal is to increase quality of life for the individuals and their caregivers (10).

\section{Drug treatment}

Drug options to help with memory loss include cholinesterase inhibitors and memantine, which treat cognitive symptoms (memory loss, confusion, etc.) (10). These medications may not prevent the degradation of neuronal cells, but they can slow the speed at which they degenerate (10). They are usually well tolerated by patients, and side effects may include nausea, vomiting, and loss of appetite (10). The stage of disease will determine the dosage and type of medication prescribed (10).

\section{Behavioral treatment}

Behavioral treatment addresses behavior problems that may arise, such as irritability, anxiety, and depression (7). These symptoms may lead to difficulties caring for an individual with Alzheimer's disease, especially for the caregiver (7). Avoiding drastic changes is an imperative factor of behavioral treatment (7). Change can be stressful and increase the individual's fear and frustration as they are trying to make sense of the situation with their impaired cognitive function (7). Situations that may trigger frustration include moving to a new residence, admission to hospitals, or being asked to alter appearance (7). Sometimes, medications can increase these symptoms of fear and anxiety (7). As a caregiver, behavioral treatment can include avoiding confrontation with the individual, redirecting their attention, creating a calm environment, and allowing the individual adequate rest (7). These behaviors can not only prevent triggering episodes but also make the individual feel more at home. There are also medications that can assist with behavior modification if behavioral treatment is ineffective, including antidepressants for low mood, antipsychotics for delusions and aggression, and anxiolytics for anxiety or restlessness (7). The use of antipsychotics for Alzheimer's disease is a very hazardous option, as it has been associated with an increased risk of stroke and death in older adults with dementia (7).

\section{MEMORY}

Our memories define our character and have a completely unique perspective than everyone else's experiences. Creating a memory involves three stages. The first, encoding, occurs when a stimulus results in the formation of a new memory (11-13). This new formation is often referred to as an engram, which is thought to be a physical memory trace in the brain (14). This trace is very susceptible to decay until the next stage occurs, consolidation (14). Consolidation is the process in which a memory becomes stable and is assimilated into previously acquired knowledge (14). The final stage, retrieval, occurs when the memory is recollected (14). 
Sometimes when we create a memory, it only lasts for a short period of time, when other memories last a lifetime $(15,16)$. Memories are temporally defined as long term or short term depending on the length of time the memory lasts. Shortterm memories last less than $2 \mathrm{~min}$, whereas long term memories can last 2 min to a lifetime $(15,16)$. Short-term memories usually are comprised of working memory $(15,16)$. Working memory typically involves small amounts of information that we only need to retain for a couple seconds, for example, the prices two items when comparing costs while shopping.

\section{Types of memory}

There are multiple types of memory and subdivisions within each type. The main two types are explicit (declarative) and implicit (nondeclarative) (15). When a memory is explicit, the individual is consciously aware of the memory, whereas with implicit memory, the individual is not (15). There are two subsets within explicit memory: episodic and semantic. Episodic, or autobiographical memories, include a what, where, and when aspect of the memory (16-18). Semantic memories are facts about the world around us (15). An example of semantic memory may be knowing that baseball is a sport, whereas an episodic memory may be remembering the first time you went to a baseball game. Implicit memories also have two subdivisions: procedural and priming (15). Procedural memories include motor skills and other actions we complete automatically without conscious thought (e.g., walking and writing) (15). Priming occurs when an individual is exposed to a stimulus that influences their response to a later stimulus (15). An example of priming may be seeing a flash of an image on a computer while taking a computer-based memory task.

\section{Forgetting}

Why are some memories retained yet others are lost? There are many reasons that we forget information we have learned or events we have experienced (19-21). The act of forgetting can occur actively or passively. Passive forgetting occurs through natural decay, or biological degradation, of neurons within a memory engram (19). Partial decay of an engram can make it challenging to activate the memory during retrieval (19). Active forgetting can occur through several mechanisms: interference, motivated forgetting, or retrieval-induced forgetting (19). Interference, which will be detailed later in this chapter, occurs when competing information makes it difficult to retrieve the correct memory (19). Motivated forgetting often occurs when an individual actively suppresses a memory due to some unpleasant quality (e.g., guilt, shame, and embarrassment) (19). Finally, retrieval-induced forgetting occurs when only parts of a memory are normally recalled, causing the other parts to degrade over time (19).

\section{Memory Interference}

As stated in the previous section, memory interference (MI) is a cause of forgetting. There are two types of MI, proactive and retroactive. Proactive interference (PI) 
occurs when previously acquired information interrupts the recall of newly learned information (old $\rightarrow$ new). For example, calling your new boyfriend by your old boyfriend's name. Retroactive interference (RI) occurs in the opposite direction, newly acquired information interrupts the recall of old information (old $\leftarrow$ new). Following the previous example, this would be calling your old boyfriend by your new boyfriend's name. Interference can be benign to serious memory disruption depending on the situation. Interference is also linked to similarity of the content. If the competing material is similar, interference is more likely to occur.

In experiments, MI is measured using paired associate learning tasks. These tasks are typically comprised of lists of word pairs or figure pairs (e.g., "bread knife" or \$) that a participant is asked to memorize as a pair. Research participants memorize lists of the word or figure pairs and subsequently recall them (e.g., bread - _ or o _ _ _ ). There are multiple models of paired associate tasks, but ones commonly used include $\mathrm{AB}-\mathrm{CD}, \mathrm{AB}-\mathrm{AC}, \mathrm{AB}-\mathrm{ABr}$, and $\mathrm{AB}-\mathrm{DE}$ $A C-F G$. For the models, the letter pairs (e.g., $A B$ and $C D$ ) signify one list each (e.g., $A B=$ List $1, C D=$ List 2), each letter in the name (e.g., $A B-C D$ ) stands for one word $(A=$ bread $B=$ knife $)$, and the combined letters (e.g., $A B)$ represent one word-pair (breadknife). Examples of measuring MI using various models are summarized in tables $1-4$.

\section{Alzheimer's disease and MI}

Alzheimer's disease causes the decay of neurons which eventually leads to memory impairment $(1,4,5,9)$. Although there are a lot of research fields focusing on how Alzheimer's disease damages memory, there is less research focusing directly on MI effects on patients with Alzheimer's disease.

When investigating patients with Alzheimer's disease and those with mild cognitive impairments without Alzheimer's disease, Dewer et al. found that memory retention is much higher in these patients when there is minimal interference compared to a normal MI paradigm (22). Their findings align with previous literature

\section{TABLE $1 \quad$ AB-CD example}

\begin{tabular}{ll} 
List $\mathbf{1}(\mathbf{A B})$ & List $\mathbf{2}(\mathbf{C D})$ \\
\hline BABY HUNTER & SPIDER CANDLE \\
SUPPER SHERIFF & ARROW THEATER \\
WEDDING MOVIE & CHERRY MONEY \\
APPLE DIAMOND & TIGER HOTEL \\
MONKEY GARDEN & CANNON HAMMER \\
FOREST BATTLE & LADY BUTTER
\end{tabular}

In this model, $\mathrm{AB}$ signifies the first list and $\mathrm{CD}$ the second list. There are no repeating letters in the title of the model, meaning there are no repeating words within the lists. Each list consists of unique words with no overlap. Participants may be exposed to both lists (learn List 1 then List 2) and then asked to recall only one of them. To measure PI, the participants will learn List 1 (AB), List 2 (CD), then recall List 2. For RI, the participants will learn List 1 (AB), List 2 (CD), then recall List 1 


\section{TABLE $2 \quad$ AB-AC example}

\begin{tabular}{ll}
\hline List $\mathbf{1}(\mathbf{A B})$ & List $\mathbf{2}(\mathbf{A C})$ \\
\hline BABY HUNTER & MOVIE WEDDING \\
SUPPER SHERIFF & APPLE CANNON \\
MONKEY GARDEN & BABY SALAD \\
FOREST BATTLE & MONKEY ENGINE \\
MOVIE CHERRY & FOREST CITY \\
APPLE TIGER & SUPPER JACKET
\end{tabular}

Similar to the previous model AB-CD, this model's first list (AB) has no repeating words, but as we can see "AC" has a repeating " $\mathrm{A}$ " word. This signifies that the "A" words from List $\mathrm{l}(\mathrm{AB})$ and List 2 (AC) will repeat, while the "B" and "C" words will not. When testing for PI, the participant will learn List 1, List 2, and then recall List 2. For RI, the participant will learn List 1, List 2, and then recall List 1.

\section{TABLE 3}

\begin{tabular}{ll}
\hline List $\mathbf{1}(\mathbf{A B})$ & List $\mathbf{2}(\mathbf{A B r})$ \\
\hline BABY HUNTER & SHERIFF CHERRY \\
SUPPER SHERIFF & DIAMOND BABY \\
WEDDING MOVIE & BATTLE SUPPER \\
SPIDER CANDLE & FOREST HUNTER \\
MONKEY GARDEN & MONEY MOVIE \\
FOREST BATTLE & SPIDER GARDEN \\
CHERRY MONEY & APPLE WEDDING \\
APPLE DIAMOND & CANDLE MONKEY
\end{tabular}

This model repeats all of the words from List $1(\mathrm{AB})$ in the second list $(\mathrm{ABr})$ except the words are rearranged into new word pairs. To reiterate, List 1 and List 2 comprised of the same words, but the way in which they are organized is different for List 2. This model can cause severe interference since the words are so similar. Participants learn List 1 , List 2, then recall either List 1 or List 2 depending on the interference being measured.

demonstrating that memory dysfunction in patients with Alzheimer's disease is associated with an increased susceptibility to MI (22). The authors hypothesize that this may be due to a decline in the ability to consolidate new memories (22). The interference paradigm utilized in this experiment is strong at predicting which patients with mild cognitive impairments will or will not progress to Alzheimer's disease within 2 years, with $80 \%$ sensitivity and 100\% specificity (22).

In term of semantic memory, or facts about the world (e.g., baseball is a sport), patients with Alzheimer's disease perform worse on these memory tasks, which may be due to a deficit in working memory and attention (23). As stated previously, working memory is short-term memory that lasts for a very short period of time with a concurrent interfering stimulus. Hartman describes how, in her experiment, there was no evidence that the patients with Alzheimer's disease utilized 


\section{TABLE 4}

\section{MMFR}

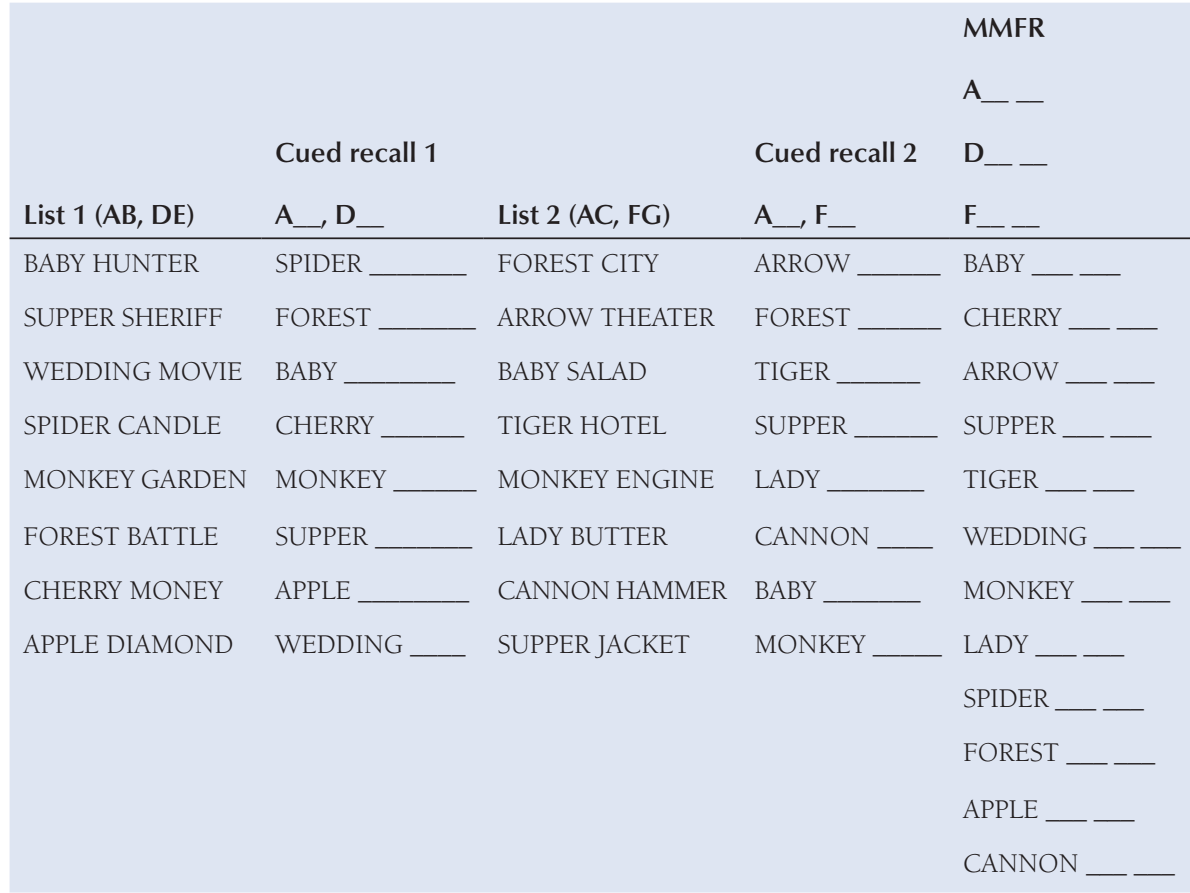

This paired associate task is more complex than the other designs because it includes control word pairs within each list, allowing for the measurement of proactive and RI within the same experiment. As before, List 1 (AB-DE) has repeating "A" words as List 2 (AC-FG). In this case, DE and FG are the control word pairs and AB and AC are the interfering word pairs. To use this model for an experiment, participants learn List 1, recall it, learn List 2, recall it, then recall the Modified Modified Free Recall (MMFR) list which is comprised of all of the word pairs from List 1 and List 2 in a pseudorandomized order.

semantic knowledge (relatedness) of word pairs during recall (23). When patients' working memory is impaired, as is typical in Alzheimer's disease, it is more difficult to retain relevant information about the relationships of words in paired associate tasks (23). Despite this experiment's focus on working memory, its results shed light on MI impairments since semantically relating words is a typical strategy utilized when memorizing word pairs in paired associate tasks. When detailing the symptoms of Alzheimer's disease, we noted that difficulty remembering and following instructions is common (23). This may also influence performance on memory tasks. Repeatedly needing external cues or verbal instructions may alter outcome scores, as mentioned elsewhere (23). Another study that focused specifically on proactive and RI compared mildly demented Alzheimer's disease patients, patients with mild cognitive impairment without Alzheimer's disease, and healthy elderly patients on interference tasks (24). When controlling for 
overall memory impairment, mild Alzheimer's disease patients demonstrated higher rates of PI, but equal amounts of RI when compared to the cognitively impaired patients (24). As expected, the healthy elderly participants experienced the least amount of interference (24). Vulnerability to semantic interference may reflect early signs of the onset of Alzheimer's disease (24).

\section{EXERCISE AND ALZHEIMER'S DISEASE}

As stated previously, physical inactivity is a risk factor for developing Alzheimer's disease and is even considered the highest population attributable risk (25). In one systematic review, the majority of experiments demonstrated that physical activity was inversely associated with risk of developing Alzheimer's disease (26). Exercise has also been demonstrated to improve multiple types of memory, including longterm and short-term memory (27-33). Exercise may even prevent the onset of Alzheimer's disease by decreasing the risk of cardiovascular disease, increasing cerebral blood flow, increasing hippocampal volume, and improving neurogenesis (34). Higher levels of physical activity are associated with a reduced risk of developing the disease (34), with long-term prospective studies demonstrating that walking regularly is associated with a twofold reduced risk in cognitive impairment (25).

During exercise, many chemicals are released in the body, including brainderived neurotrophic factor, which is directly associated with learning and memory (35). Research also demonstrates that regular physical activity prevents mental decline and improves thinking in populations with vascular cognitive impairment (34). It has been used clinically in the treatment of preclinical and late-stage Alzheimer's disease, as well as a prevention strategy (34). Recent prospective work, that compared sedentary individuals to those who were the most active, demonstrated a 38\% reduction in incidence of Alzheimer's disease (36). In animal studies, mice with Alzheimer's disease that completed 16 weeks of treadmill exercise have been shown to elicit changes in therapeutic parameters at the cellular and molecular level, providing biological plausibility to exercise as a therapy (37).

In order to reduce the risk of developing Alzheimer's disease, to lessen the effects for those already suffering from memory loss, individuals should participate in regular physical activity. The American national guidelines suggest at least 150 min per week of moderate to vigorous physical activity (38). Meta-analyses have demonstrated mixed findings on which mode of exercise is best for improving specific types of memory; however, walking, cycling, and jogging are three of the most common exercises implemented, all of which have demonstrated beneficial effects $(33,39)$. Multicomponent exercise programs have also been effective in improving cognitive function in institutionalized older adults with mild to moderate Alzheimer's disease (40). One particular study found that incorporating a program that included supervised aerobic, muscular resistance, flexibility, and postural exercises for 45-55 min sessions twice per week for 6 months significantly improved patients' cognitive function when compared to a control group (40). These findings suggest that incorporating a variety of physical activities may be an effective non-pharmacological method for improving cognitive function, along with physical function, in those with Alzheimer's disease (40). 


\section{CONCLUSION}

In summary, individuals with Alzheimer's disease may be more susceptible to MI, due to dysfunction of working memory and semantic memory. This can cause even more confusion, as patients already cope with a myriad of memory problems and other symptoms. The literature on Alzheimer's disease and MI is sparse, and therefore, it is imperative that the field continues to grow and search for methods to attenuate interference in this population - for example, through exercise, which has been demonstrated to increase memory performance. Experiencing MI, or difficulty recalling information because of competing memories, can be very frustrating and debilitating for anyone, but especially for patients with Alzheimer's disease. In experimental settings, paired associate tasks are often utilized to measure interference, but there are also other methods that are suitable. Due to the debilitating nature of Alzheimer's disease, it is important to focus on prevention and delaying the condition by keeping your mind and body active, eating a healthy diet, and wearing safety equipment to avoid head injury.

Conflict of interest: The authors declare no potential conflicts of interest with respect to research, authorship, and/or publication of this chapter.

Copyright and Permission Statement: To the best of our knowledge, the materials included in this chapter do not violate copyright laws. All original sources have been appropriately acknowledged and/or referenced. Where relevant, appropriate permissions have been obtained from the original copyright holder(s).

\section{REFERENCES}

1. Aging NIo. Alzheimer's disease \& related dementias [Internet]. Available from: https://www.nia.nih. gov/health/alzheimers/basics

2. Association As. What is Alzheimer's? [Internet]. Available from: https://www.alz.org/alzheimers-dementia/ what-is-alzheimers\#basics

3. Association As. Younger/early onset [Internet]. Available from: https://www.alz.org/alzheimersdementia/what-is-alzheimers/younger-early-onset

4. Clinic M. Alzheimer's disease [Internet]. Available from: https://www.mayoclinic.org/diseases-conditions/alzheimers-disease/symptoms-causes/syc-20350447

5. Association As. 10 early signs and symptoms of Alzheimer's [Internet]. Available from: https://www. alz.org/alzheimers-dementia/10_signs

6. Association As. Stages of Alzheimer's [Internet]. Available from: https://www.alz.org/alzheimers-dementia/ stages

7. Association As. Treatments for behavior [Internet]. Available from: https://www.alz.org/alzheimers-dementia/ treatments/treatments-for-behavior

8. Association As. Causes and risk factors [Internet]. Available from: https://www.alz.org/alzheimers-dementia/ what-is-alzheimers/causes-and-risk-factors

9. Medicine PCfR. Alzheimer's disease [Internet]. Available from: https://www.pcrm.org/health-topics/ alzheimers

10. Association As. Medications for memory [Internet]. Available from: https://www.alz.org/ alzheimers-dementia/treatments/medications-for-memory

11. Puglisi JT, Park DC, Smith AD, Dudley WN. Age differences in encoding specificity. J Gerontol. 1988;43(6):P145-50. http://dx.doi.org/10.1093/geronj/43.6.P145 
12. Stickgold R, Walker MP. Sleep-dependent memory consolidation and reconsolidation. Sleep Med. 2007;8(4):331-43. http://dx.doi.org/10.1016/j.sleep.2007.03.011

13. McGaugh JL. Memory - A century of consolidation. Science. 2000;287(5451):248-51. http://dx.doi. org/10.1126/science.287.5451.248

14. Loprinzi PD, Edwards MK, Frith E. Potential avenues for exercise to activate episodic memory-related pathways: A narrative review. Eur J Neurosci. 2017;46(5):2067-77. http://dx.doi.org/10.1111/ ejn. 13644

15. Institute QB. Types of memory The University of Queensland 2018, July 23 [Internet]. Available from: https://qbi.uq.edu.au/brain-basics/memory/types-memory

16. Moscovitch M, Cabeza R, Winocur G, Nadel L. Episodic memory and beyond: The hippocampus and neocortex in transformation. Annu Rev Psychol. 2016;67:105-34. http://dx.doi.org/10.1146/ annurev-psych-113011-143733

17. Scully ID, Napper LE, Hupbach A. Does reactivation trigger episodic memory change? A meta-analysis. Neurobiol Learn Mem. 2017;142(Pt A):99-107. http://dx.doi.org/10.1016/j.nlm.2016.12.012

18. Shields GS, Sazma MA, McCullough AM, Yonelinas AP. The effects of acute stress on episodic memory: A meta-analysis and integrative review. Psychol Bull. 2017;143(6):636-75. http://dx.doi.org/10.1037/ bul0000100

19. Davis RL, Zhong Y. The biology of forgetting - A perspective. Neuron. 2017;95(3):490-503. http:// dx.doi.org/10.1016/j.neuron.2017.05.039

20. Aguirre C, Gomez-Ariza CJ, Andres P, Mazzoni G, Bajo MT. Exploring mechanisms of selective directed forgetting. Front Psychol. 2017;8:316. http://dx.doi.org/10.3389/fpsyg.2017.00316

21. Anderson MC, Hanslmayr S. Neural mechanisms of motivated forgetting. Trends Cogn Sci. 2014;18(6):279-92. http://dx.doi.org/10.1016/j.tics.2014.03.002

22. Dewar M, Pesallaccia M, Cowan N, Provinciali L, Della Sala S. Insights into spared memory capacity in amnestic MCI and Alzheimer's disease via minimal interference. Brain Cogn. 2012;78(3):189-99. http://dx.doi.org/10.1016/j.bandc.2011.12.005

23. Hartman M. The use of semantic knowledge in Alzheimer's disease: Evidence for impairments of attention. Neuropsychologia. 1991;29(3):213-28. http://dx.doi.org/10.1016/0028-3932(91)90083-K

24. Loewenstein DA, Acevedo A, Luis C, Crum T, Barker WW, Duara R. Semantic interference deficits and the detection of mild Alzheimer's disease and mild cognitive impairment without dementia. J Int Neuropsychol Soc. 2004;10(1):91-100. http://dx.doi.org/10.1017/S1355617704101112

25. Loprinzi PD, Frith E, Ponce P. Memorcise and Alzheimer's disease. Phys Sports Med. 2018;46(2): 145-54. http://dx.doi.org/10.1080/00913847.2018.1445932

26. Norton S, Matthews FE, Barnes DE, Yaffe K, Brayne C. Potential for primary prevention of Alzheimer's disease: An analysis of population-based data. Lancet Neurol. 2014;13(8):788-94. http://dx.doi. org/10.1016/S1474-4422(14)70136-X

27. Angevaren M, Aufdemkampe G, Verhaar HJ, Aleman A, Vanhees L. Physical activity and enhanced fitness to improve cognitive function in older people without known cognitive impairment. Cochrane Database Syst Rev. 2008;(3):CD005381. http://dx.doi.org/10.1002/14651858.CD005381.pub3

28. Chang YK, Labban JD, Gapin JI, Etnier JL. The effects of acute exercise on cognitive performance: A meta-analysis. Brain Res. 2012;1453:87-101. http://dx.doi.org/10.1016/j.brainres.2012.02.068

29. Colcombe S, Kramer AF. Fitness effects on the cognitive function of older adults: A meta-analytic study. Psychol Sci. 2003;14(2):125-30. http://dx.doi.org/10.1111/1467-9280.t01-1-01430

30. Cotman CW, Berchtold NC. Exercise: A behavioral intervention to enhance brain health and plasticity. Trends Neurosci. 2002;25(6):295-301. http://dx.doi.org/10.1016/S0166-2236(02)02143-4

31. McMorris T, Hale BJ. Differential effects of differing intensities of acute exercise on speed and accuracy of cognition: A meta-analytical investigation. Brain Cogn. 2012;80(3):338-51. http://dx.doi. org/10.1016/j.bandc.2012.09.001

32. McMorris T, Sproule J, Turner A, Hale BJ. Acute, intermediate intensity exercise, and speed and accuracy in working memory tasks: A meta-analytical comparison of effects. Physiol Behav. 2011;102(3-4):421-8. http://dx.doi.org/10.1016/j.physbeh.2010.12.007

33. Roig M, Nordbrandt S, Geertsen SS, Nielsen JB. The effects of cardiovascular exercise on human memory: A review with meta-analysis. Neurosci Biobehav Rev. 2013;37(8):1645-66. http://dx.doi. org/10.1016/j.neubiorev.2013.06.012 
34. Cass SP. Alzheimer's disease and exercise: A literature review. Curr Sports Med Rep. 2017;16(1): 19-22. http://dx.doi.org/10.1249/JSR.0000000000000332

35. Loprinzi PD, Frith E. A brief primer on the mediational role of BDNF in the exercise-memory link. Clin Physiol Funct Imaging. 2019;39(1):9-14. http://dx.doi.org/10.1111/cpf.12522

36. Guure CB, Ibrahim NA, Adam MB, Said SM. Impact of physical activity on cognitive decline, dementia, and its subtypes: Meta-analysis of prospective studies. Biomed Res Int. 2017;2017:9016924. http://dx.doi.org/10.1155/2017/9016924

37. Um HS, Kang EB, Leem YH, Cho IH, Yang CH, Chae KR, et al. Exercise training acts as a therapeutic strategy for reduction of the pathogenic phenotypes for Alzheimer's disease in an NSE/APPswtransgenic model. Int J Mol Med. 2008;22(4):529-39.

38. Piercy KL, Troiano RP, Ballard RM, Carlson SA, Fulton JE, Galuska DA, et al. The physical activity guidelines for Americans. JAMA. 2018;320(19):2020-8. http://dx.doi.org/10.1001/jama.2018.14854

39. Loprinzi PD, Blough J, Crawford L, Ryu S, Zou L, Li H. The temporal effects of acute exercise on episodic memory function: Systematic review with meta-analysis. Brain Sci. 2019;9(4):pii: E87. http:// dx.doi.org/10.3390/brainsci9040087

40. Sampaio A, Marques EA, Mota J, Carvalho J. Effects of a multicomponent exercise program in institutionalized elders with Alzheimer's disease. Dementia (London). 2019;18(2):417-31. http://dx.doi. org/10.1177/1471301216674558 